\section{Characterization of Puncture Forces for Retinal Vein Cannulation}

\author{
Olgaç Ergeneman \\ e-mail: oergeneman@ethz.ch
}

\section{Juho Pokki}

\section{Vanda Počepcová}

Institute of Robotics and Intelligent Systems, ETH Zurich, 8092 Zurich, Switzerland

\section{Heike Hall}

Cells and BioMaterials,

Department of Materials,

ETH Zurich, 8093 Zurich, Switzerland

\author{
Jake J. Abbott \\ Department of Mechanical Engineering, \\ University of Utah, \\ Salt Lake City, Utah, 84112 \\ Bradley J. Nelson \\ Institute of Robotics and Intelligent Systems, \\ ETH Zurich, 8092 Zurich, Switzerland
}

For this study, we have collected puncture force data from the vasculature of the chorioallantoic membranes (CAM) of developing chicken embryos to examine forces required for retinal vein cannulation. The CAM vessels of a developing chicken embryo have been shown to be an appropriate model for human retinal veins. The effect of microneedle geometry and vessel size on puncture forces was investigated. The results of this work are important for researchers working on robotic vitreoretinal surgical systems. [DOI: 10.1115/1.4005318]

\section{Introduction}

Retinal diseases such as age-related macular degeneration, diabetic retinopathy, and retinal vein occlusion (RVO) are major causes of vision impairment and blindness in industrial countries [1-3]. Microsurgical interventions in the posterior eye segment are part of the therapeutical management of these diseases. Retinal microsurgery requires complex and sensitive manipulation at the limits of human performance [4]. Forces applied by the surgeon are mostly below the threshold of human perception [5], providing strong evidence that manual retinal microsurgery relies almost exclusively on visual feedback. These interventions set high demands on the precision of the surgical instruments as well as the control of forces [6-8].

Various robotic surgical systems for ophthalmology aim to improve positioning capability for microsurgeons, such as the "Steady Hand" cooperative robotic system $[9,10]$, and the handheld active tremor-canceling device, "Micron" [11]. Recently, a robotic system for intraocular dexterity enhancement was proposed [12]. Each of the above-mentioned systems utilizes a robot-assisted surgical instrument that passes through a hole in the sclera. As an alternative to the mentioned robotic surgical systems, a wireless magnetic microrobot for ophthalmic procedures

Manuscript received March 29, 2011; final manuscript received September 2, 2011; published online November 15, 2011. Assoc. Editor: Paul A. Iaizzo. has been proposed to ensure the accuracy of the performed surgery and to potentially avoid the necessity of vitrectomy [13]. The microrobot is to be inserted into the eye by a syringe and wirelessly steered to the target area by external magnetic fields generated by a magnetic manipulation system [14]. The microrobot together with the navigation system can find applications in the therapy of diverse ophthalmic disorders.

Robotic surgical systems can be particularly important for the treatment of RVO. RVO is a common vascular disease (cumulative incidence $2.3 \%$ ) and may lead to severe vision impairment [1]. The primary methods for treating RVO entail systemic and intravitreal drug administration. Surgical procedures, such as injection of tPA into branch retinal veins, are used in more extreme cases $[15,16]$. This enables administration directly into the affected vein; hence, it requires lower concentrations of tPA. Retinal veins are small, delicate, and thin-walled structures. Prolonged manual cannulation of retinal veins risks causing permanent damage to the retina due to the tremor and physical fatigue of the surgeon. A robotic system can assist the cannulation process to increase the accuracy of the procedure. A microrobot can be employed as a microscopic syringe that is able to deliver tPA directly into a clot within a retinal vein.

A quantitative study on the magnitude of forces needed to puncture retinal veins is not available in the literature. In Ref. [4], retinal cannulation forces, together with scleral interaction forces, are measured. However, needle and blood-vessel size, which affect puncture forces, are not specified. In Ref. [5], a retinal pick equipped with strain gauges is used to manipulate the retina of porcine cadaver eyes, and the range of forces acquired throughout a typical procedure is reported. However, the force of an individual retinal vein cannulation is not provided.

Conducting in vivo experiments on animal eyes is difficult with a high risk of tissue damage, and postmortem experiments may provide inaccurate results due to changes in tissue properties of vessels after death. To examine forces required for retinal vein cannulation, we have performed experiments with chicken embryos. Puncture force data are collected from the vasculature of the chorioallantoic membrane (CAM) of a developing chicken embryo, which has been shown to be an appropriate model for human retinal vessels [17]. The effect of microneedle geometry and vessel size on puncture forces was investigated. Knowledge of forces necessary for retinal punctures is important for researchers working on the design and control of intraocular microrobots, robot-assisted retinal vein cannulation systems, and microsurgical instruments. This information can be used for setting design specifications for delicate and compliant devices [12] as well as for defining safety limits in the control systems of other rigid devices with force-sensing capabilities $[9,10]$.

\section{Materials and Methods}

2.1 Chorioallantoic Membrane and its Preparation. The chorioallantoic membrane (CAM) of developing chicken embryos has been used by ophthalmologists as a model system for studying photodynamic therapy and ocular angiogenesis. Recently, it was reported that the CAM of a 12-day-old chicken embryo is a valid in vivo test system for studies on human retinal vein cannulation $[10,17]$. The CAM's anatomical features and physiologic and histologic responses to manipulation and injury make it an effective model of the retina and its vasculature. The vasculature of a twelve-day-old CAM and a human retina have roughly the same diameter and wall thickness (i.e., blood vessels with 50-400 $\mu \mathrm{m}$ outer diameter).

In this study, the CAMs were prepared as described in Ref. [17]. Fertilized chicken eggs were bred in incubators at $37^{\circ}$ and approximately $50 \%$ relative humidity. The eggs were kept in the incubator before using them in the experiments, with a yield of incubation about $80 \%$. Shortly before the experiments, CAMs inside their egg shells were transferred into petri dishes. For this, a cut to the egg shell was made carefully with a conical cutting 


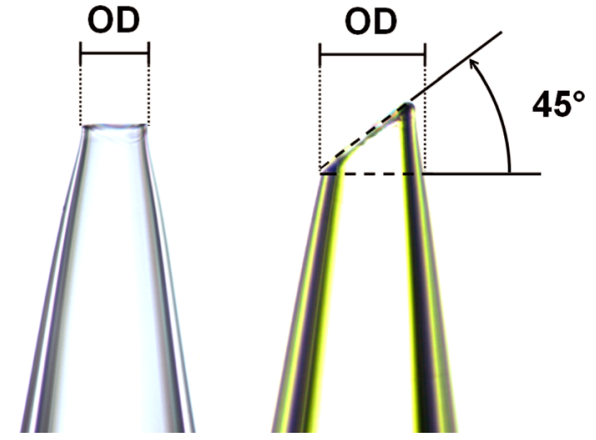

Fig. 1 Two types of microneedles were prepared: blunt and beveled. The outer diameter (OD) and bevel angle is shown in the image.

tool and the CAMs were transferred, paying attention not to break the yolk.

2.2 Microneedle Preparation. Two types of microneedles as blunt tips and beveled tips (Fig. 1) were prepared with five different outer diameters (OD). All microneedles were prepared with a micropipette puller (Sutter Instruments, P-2000 Laser-based Micropipette Puller) using hollow borosilicate glass capillaries (Harvard apparatus, GC100-10, OD $1.0 \mathrm{~mm}$, inner diameter $0.58 \mathrm{~mm}$ ). The blunt tips were used without further treatment. The beveled microneedles were polished using a micropipette beveler (World Precision Instruments SYS-48000). The bevel angle used was $45^{\circ}$. The needle tip ODs and bevel angles were verified using an optical microscope.

2.3 Measurement Setup. The setup for the puncture experiments is shown in Fig. 2. A microscope (Olympus SZX9) with a digital camera (Canon PowerShot A640) was utilized for imaging and video acquisition in order to observe the puncture event, and to image the blood vessels punctured. The microneedle was mounted on the force sensor with its axis aligned along the direction of sensing. The force sensor (Picodyne, MN, USA) measured the force applied by the microneedle to a blood vessel. The sensor is insensitive to torques. The force sensor with the microneedle was placed on the 3-degree-of-freedom micromanipulator (Sutter Instruments, MP-285), which allowed the needle to advance toward a blood vessel at a controlled speed. The microneedle was mounted at $45^{\circ}$ from the normal of the plane of the petri dish and was advanced in its axial direction. The force sensor output was monitored in real-time using a DAQ card and recorded with a time stamp using a custom-developed program (Labview 9.0, National Instruments). The sensor output was sampled at $10 \mathrm{kHz}$ and averaged to reduce noise.

2.4 Calibration of the Force Sensor. The force sensor was calibrated by the manufacturer (Picodyne, MN, USA). The orientation of the sensor does not affect the sensor calibration. The gain of the force sensor was verified before and after each set of experiments using custom weights. The custom weights were measured using a Mettler Toledo XS 204 analytical balance, which is traceable to international standards. No significant variation in the gain (i.e., smaller than $0.5 \%$ ) was observed throughout the experiments. The force sensor has a resolution smaller than $2 \mu \mathrm{N}$ at a $1 \mathrm{kHz}$ sampling rate. The weight of the attached microneedle creates an offset in the force output. As the weight of the microneedle is within the usable range of the force sensor and the sensor is insensitive to torques, the calibration was valid over the entire duration of the experiment.

2.5 Experimental Procedure. CAMs were prepared and transfered into the petri dish as described in Sec. 2.1. Phosphate

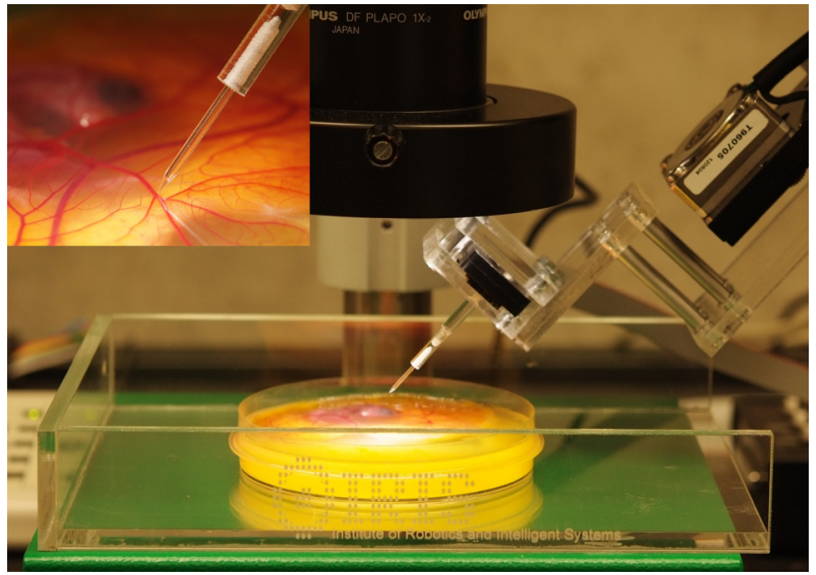

Fig. 2 Setup for puncture-force experiments. A microneedle is attached on a force sensor, which is mounted on a micromanipulator. The puncture events were observed using a microscope and images were captured by a camera. On the top left, a microneedle advancing toward a blood vessel is shown. The microneedle was mounted at $45^{\circ}$ from the normal of the plane of the petri dish and was advanced in its axial direction. The petri dish was oriented to have the vessel axis perpendicular to the microneedle axis.

buffer solution (PBS, pH 7.4, Sigma-Aldrich) was applied to the CAM in order to keep the surface moist and to improve visualization through the microscope during the experiments. A blood vessel on the CAM was chosen randomly using the following criteria: (1) the vessel was attached to the yolk so that it could be punctured without complex fixation, and (2) the vessel had a roughly constant $\mathrm{OD}$ over a minimum length of $2 \mathrm{~mm}$. The blood vessel was imaged using the microscope and the digital camera in order to determine vessel OD. The force data was recorded before penetrating into the CAM to correct for gravitational forces and offset of the sensor. The microneedle advanced toward the vessel with its axis perpendicular to the vessel axis and was moved to push the vessel toward its center and against its support. It was advanced at a constant speed of $55 \mu \mathrm{m} / \mathrm{s}$ with the use of the micromanipulator toward the vessel until a puncture was observed. This speed was chosen to ensure precise and controlled punctures, and it is also within the range that existing microsurgical tools are steered $[14,18]$. A puncture is detected when a drop in the realtime force data was observed and the needle was seen to penetrate into the sample. In most cases the puncture event was also verified by bleeding of the vessel.

\section{Results}

3.1 Puncture Event. In Fig. 3, force data is presented as a force-time plot showing the phases of the puncture experiment. As the speed was kept constant throughout the experiments, the force-time characteristics are analogous to force-deflection characteristics.

In Fig. 3(a), the microneedle had no contact to the CAM. Then it was moved until it touched the blood vessel to be punctured. An increase in force was observed (negative sign for compressive force).

In Fig. 3(b), the microneedle was held in place touching the vessel.

In Fig. 3(c), the microneedle was driven with a constant speed toward the blood vessel until a puncture was observed. A few seconds after the puncture, the movement of the microneedle was stopped by the operator.

In the Fig. 3(d), the microneedle remained inside the vessel tissue without moving. The magnitude of force decreased, potentially due to relaxation effects of the punctured vessel [19].

In Fig. 3(e), the microneedle was withdrawn with a constant speed; in addition, a pulling force (force with positive sign) was 


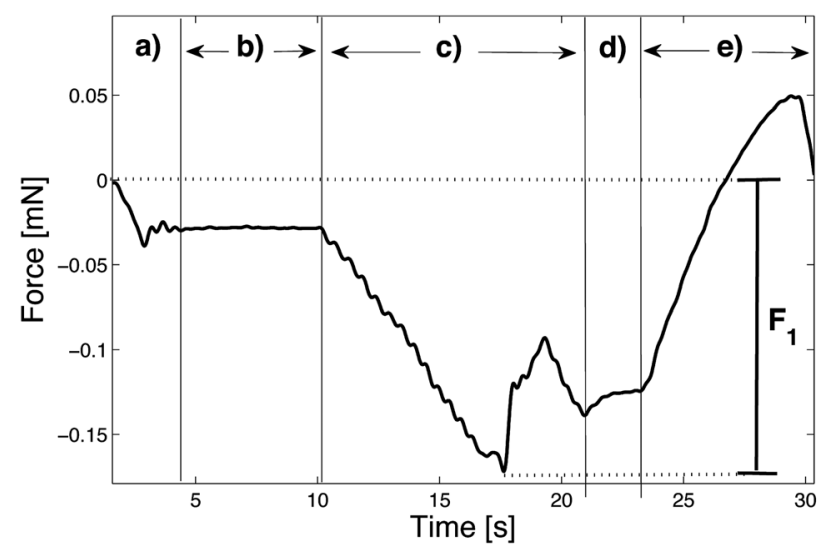

(a) Typical puncture event with 1-2 $\mu \mathrm{m}$ OD tips

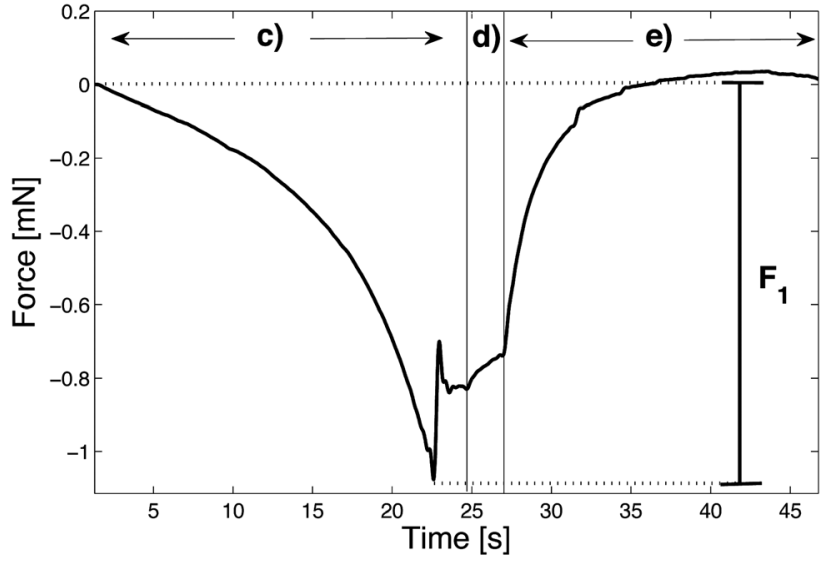

(b) Typical punctures with $\geq 10 \mu \mathrm{m}$ OD tips

Fig. 3 Puncture force $\left(F_{1}\right)$ and the phases of the puncture-force experiment are shown. The compressive force on the sensor is shown as negative force. The steps in plot (a) are due to the movement of the stepper motor, and they are not seen in (b) because the magnitude of forces is higher.

detected as the microneedle was pulled out of the blood vessel until needle and blood vessel were separated from each other. The pulling force was due to a tight seal around the needle by the elastic blood-vessel tissue.

The puncture forces $\left(F_{1}\right)$ were detected from the force-time plots. The data in Fig. 3(a) was acquired using the smallest tip OD of $1.5 \mu \mathrm{m}$. The force-time plot with this needle OD showed quasielastic behavior with a linear curve during the needle insertion phase. This behavior is described as a typical material behavior experiencing only small deformations [19]. Using microneedles with tip ODs over $10 \mu \mathrm{m}$, nonlinear behavior was observed in the force-time plots (Fig. 3(b)). This behavior is typical for vessels that show viscoelastic characteristics.

3.2 Experimental Results. A total of 459 successful puncture force measurements were performed. Table 1 gives the number of measurements for each OD of microneedle and the number of vessels punctured for blunt and beveled microneedles. The majority $(85 \%)$ of the puncture forces of all measurements were under $5 \mathrm{mN}$. This is consistent with Ref. [5] where it is reported that $63 \%$ of the forces were under $5 \mathrm{mN}$ when porcine cadaver eye blood vessels were punctured. In both studies, the majority of forces were below $2.5 \mathrm{mN}$ : $64 \%$ of forces below $5 \mathrm{mN}$ were also below $2.5 \mathrm{mN}$ in our study, and $67 \%$ of forces below $5 \mathrm{mN}$ were also below $2.5 \mathrm{mN}$ in Ref. [5].

Table 1 Puncture force measurements with blunt and beveled microneedles

\begin{tabular}{lcc}
\hline \hline Range of tip OD & Number of punctures & Number of blood vessels \\
\hline Blunt needles & & \\
$1-2 \mu \mathrm{m}$ & 53 & 9 \\
$9-15 \mu \mathrm{m}$ & 90 & 33 \\
$29-34 \mu \mathrm{m}$ & 31 & 7 \\
$46-51 \mu \mathrm{m}$ & 108 & 26 \\
$70-73 \mu \mathrm{m}$ & 24 & 7 \\
Total & 306 & 82 \\
Beveled needles & & \\
$13-15 \mu \mathrm{m}$ & 72 & 17 \\
$25 \mu \mathrm{m}$ & 27 & 7 \\
$46-52 \mu \mathrm{m}$ & 33 & 11 \\
$68-69 \mu \mathrm{m}$ & 21 & 5 \\
Total & 153 & 40 \\
\hline \hline
\end{tabular}

A histogram of different puncture forces obtained in the measurements with $1 \mathrm{mN}$ intervals is shown in Fig. 4 for blunt and beveled microneedles. Blood vessels smaller than $400 \mu \mathrm{m}$ in diameter were used to produce the data in order to avoid a bias caused by the size difference. This range covers $90 \%$ of the measurements and is of interest for retinal cannulations. Figures 5 and 6 show the puncture-force data for blunt and beveled microneedles, respectively. Each point in the figures represents the average of a group of puncture forces (minimum of three per vessel) measured from a specific blood vessel.

3.3 Regression Analysis. The dependency of the puncture force on the needle OD, the needle type, and the vessel OD was investigated by performing regression analysis on the puncture force data using R (RStudio). The puncture force was fitted to a model with the vessel OD and the needle OD as independent variables. A supplementary model, with the needle type as the third independent variable, was utilized to evaluate the effect of the needle type on the puncture force.

Average values of 3-10 puncture force measurements from each blood vessel within the range of $80-600 \mu \mathrm{m}$ were used in the regression models. The smallest microneedle set $(1-2 \mu \mathrm{m})$ was used in both models (i.e., blunt and beveled) as the effect of needle type at this size was negligible and it was difficult to prepare good quality beveled needles. The models were evaluated using

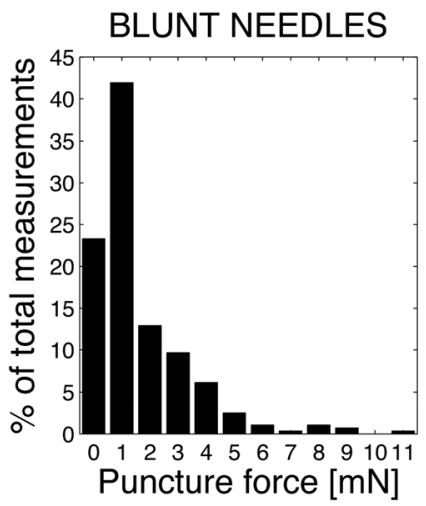

BEVELED NEEDLES

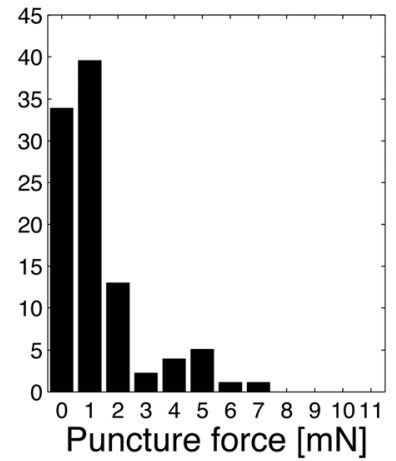

Fig. 4 Histogram of magnitude of forces as percentages of all measurements. Vessels in $80-400 \mu \mathrm{m}$ OD range were considered. 


\section{BLUNT NEEDLES}

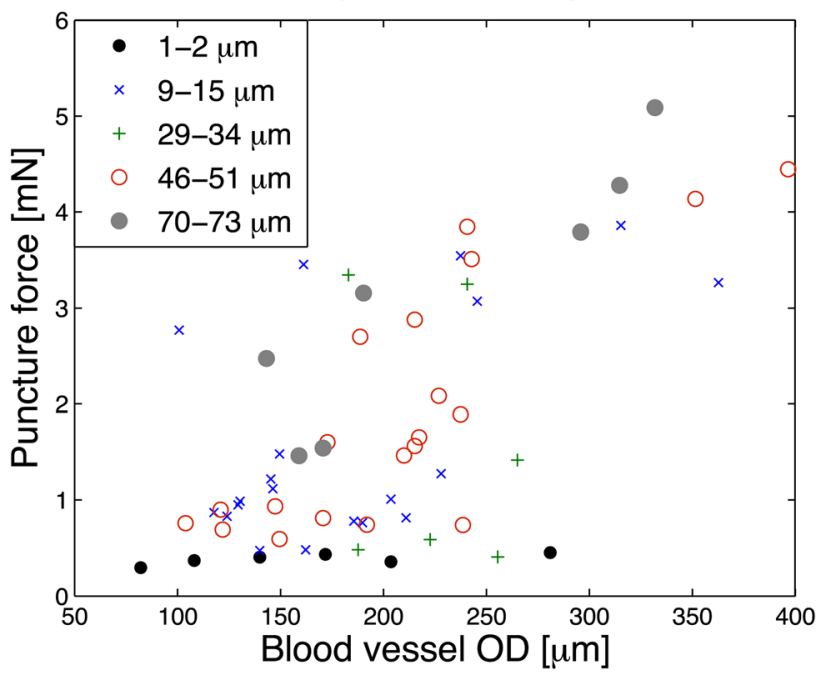

Fig. 5 Average puncture forces for 1-2 $\mu \mathrm{m}, 9-15 \mu \mathrm{m}, 29-34 \mu \mathrm{m}$, 46-51 $\mu \mathrm{m}$, and 70-73 $\mu \mathrm{m}$ tip OD for the blunt microneedles (306 individual punctures)

residual analysis and the backward selection algorithm (BSA) based on Akaike information criterion (AIC). The force data was first fitted to a quadratic polynomial using the needle OD and the vessel OD as independent variables. The residual analysis of this model showed heteroscedasticity (i.e., differing variance of the residuals). Based on the residual analysis, a logarithmic transformation of the force data $\left(F_{p}\right)$ was fitted to a quadratic polynomial (Eq. (1)). In Eq. (1), $\phi_{v}$ is the vessel OD, $\phi_{n}$ is the needle OD, and $\beta_{x x}$ are coefficients for each term.

$$
\log \left(F_{p}\right)=\beta_{00}+\beta_{01} \phi_{v}+\beta_{10} \phi_{n}+\beta_{11} \phi_{v} \phi_{n}+\beta_{02} \phi_{v}^{2}+\beta_{20} \phi_{n}^{2}
$$

The BSA was applied, and a linear polynomial was found as the optimal model for the logarithm of the puncture force data with the blunt and beveled needles (Eq. (2)).

$$
\log \left(F_{p}\right)=\beta_{00}+\beta_{01} \phi_{v}+\beta_{10} \phi_{n}
$$

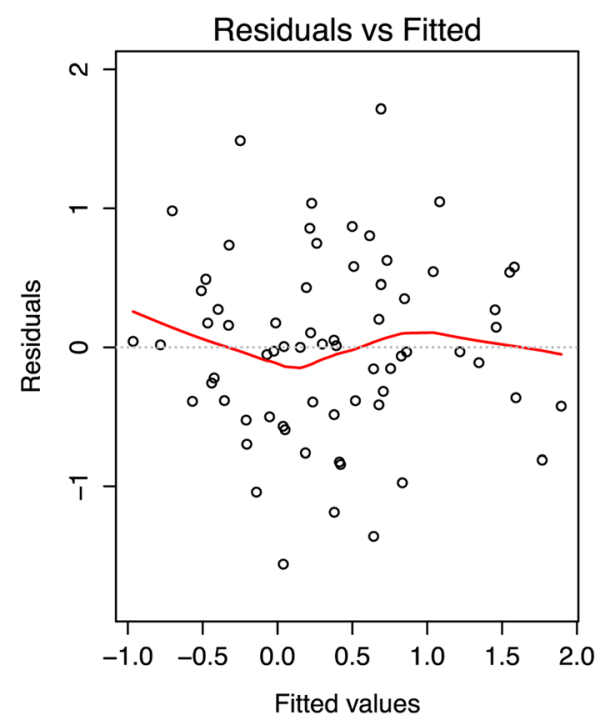

BEVELED NEEDLES

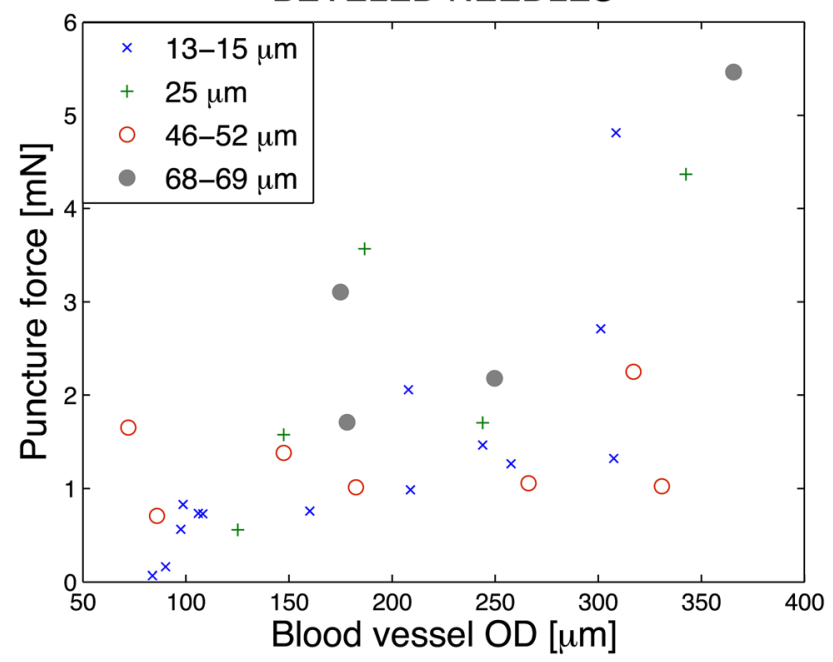

Fig. 6 Average puncture forces for $13-15 \mu \mathrm{m}, 25 \mu \mathrm{m}, 46-52 \mu \mathrm{m}$, and 68-69 $\mu \mathrm{m}$ tip OD for the beveled microneedles (153 individual punctures)

The residual analysis of this model showed homogeneous scatter of the residuals (Residuals versus Fitted and Normal Q-Q Plots) for the blunt needles (Fig. 7) and the beveled needles (Fig. 8). These models exhibited homoscedasticity with a roughly constant variance over the force range. No potential outliers or influential points were found. Using this model of the logarithmic force, the final regression model of the puncture force is an exponential equation describing the force data (Eq. (3)).

$$
F_{p}=e^{\beta_{00}+\beta_{01} \phi_{v}+\beta_{10} \phi_{n}}
$$

Table 2 presents the coefficients $\left(\beta_{x x}\right)$, their $95 \%$ confidence intervals, and their $\mathrm{p}$-values for the models with blunt and beveled needles.

The regression models indicated that the puncture forces tend to increase with respect to microneedle tip OD ( $\mathrm{p}$-value $=3 \times 10^{-4}$ for blunt needles, and $\mathrm{p}$-value $=0.0114$ for beveled needles) and with respect to vessel OD ( $p$-value $=6 \times 10^{-10}$ for blunt needles, and $\mathrm{p}$-value $=2 \times 10^{-8}$ for beveled needles). Root-mean-square

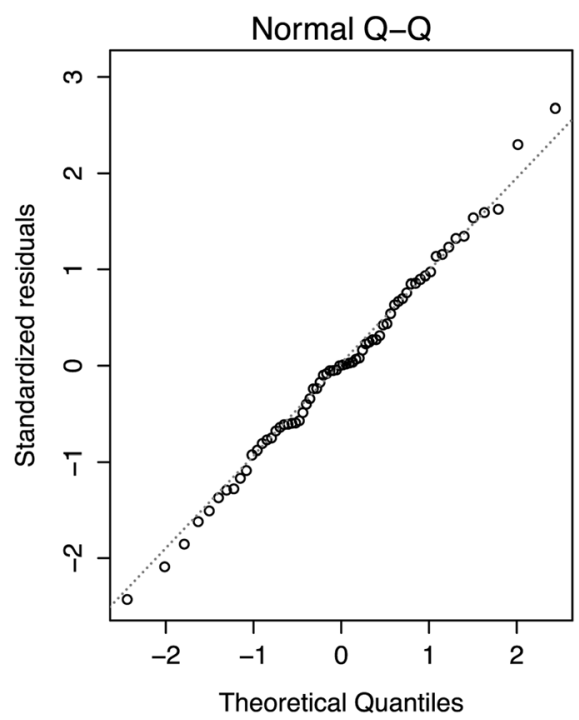

Fig. 7 Residual analysis for the regression model using the force data with blunt needles 

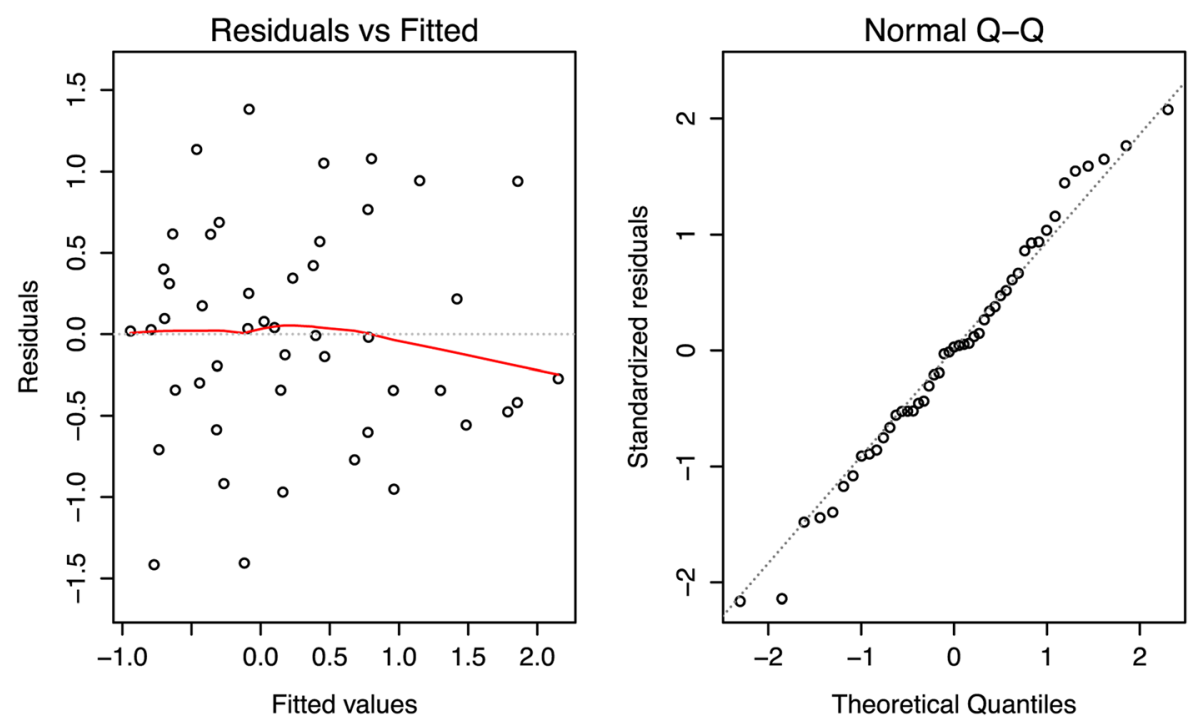

Fig. 8 Residual analysis for the regression model using the force data with beveled needles

Table 2 Model coefficients

\begin{tabular}{lcccc}
\hline \hline Coefficient & Unit & Value & $\begin{array}{c}95 \% \text { confidence } \\
\text { interval }\end{array}$ & P-value \\
\hline Blunt needles & & & & \\
$\beta_{00}$ & $\ln (\mathrm{mN})$ & -1.5407 & $-2.0322--1.0492$ & $3 \times 10^{-8}$ \\
$\beta_{01}$ & $\ln (\mathrm{mN}) / \mu \mathrm{m}$ & 0.0067 & $0.0049-0.0086$ & $6 \times 10^{-10}$ \\
$\beta_{10}$ & $\ln (\mathrm{mN}) / \mu \mathrm{m}$ & 0.0161 & $0.0076-0.0246$ & $3 \times 10^{-4}$ \\
Beveled needles & & & & \\
$\beta_{00}$ & $\ln (\mathrm{mN})$ & -1.4118 & $-1.8662-0.9574$ & $1 \times 10^{-7}$ \\
$\beta_{01}$ & $\ln (\mathrm{mN}) / \mu \mathrm{m}$ & 0.0055 & $0.0039-0.0071$ & $2 \times 10^{-8}$ \\
$\beta_{10}$ & $\ln (\mathrm{mN}) / \mu \mathrm{m}$ & 0.0120 & $0.0028-0.0212$ & $1 \times 10^{-2}$ \\
\hline \hline
\end{tabular}

deviation (RMSD) for the model with the blunt needles was $1.8 \mathrm{mN}$ and with the beveled needles was $2.1 \mathrm{mN}$. In the supplementary model with the needle type included, beveling of the needle exhibited decrease in the puncture forces with a p-value of 0.0235 .

The data have large variations (average $\sigma \simeq 35 \%$ ) due to the anatomical variances of CAM vessels and the developmental state of the CAM. The beveling of the microneedle decreased the forces necessary to puncture the vessels, especially at larger microneedle sizes (i.e., over $30 \mu \mathrm{m}$ ). The smallest microneedle size (i.e., $1-2 \mu \mathrm{m})$ gave the lowest range of forces $(0.1-0.6 \mathrm{mN})$ necessary for vessel puncture, and they did not show significant variation over the entire range of blood vessels investigated. The biological data have large variations; therefore, fitting a predictive model is challenging.

\section{Conclusion}

Puncture forces necessary for retinal vein cannulation were characterized by performing in vivo measurements on the CAM vessels of developing chicken embryos. The puncture force dependency on vessel size and microneedle tip OD and geometry was investigated. A regression analysis was carried out, and a model with homoscedastic scatter of the residuals could be found. Using this model, statistically significant effects of the vessel size, microneedle size, and microneedle type on the puncture force could be represented. The data presented is valuable for researchers working on the design and control of intraocular microrobots, robot-assisted retinal vein cannulation systems, and microsurgical instruments. Knowledge of retinal puncture forces can be used for determining specifications for such systems and also for defining safety limits.

\section{Acknowledgment}

The authors would like to thank Dr. S. Pané, Mr. K. Sivaraman, and Mr. G. Chatzipirpiridis for their support throughout this work, Dr. Z. Boutaghou for advice on experimental work, Dr. J. Kusch for help on needle preparation, Ms. Anna Drewek for her input to the statistical analysis, and Professor P. Vögeli for the use of their incubator (all from ETH Zurich). This work is supported by the NCCR Co-Me of the Swiss National Science Foundation.

\section{References}

[1] Klein, R., Moss, S. E., Meuer, S. M., and Klein, B. E. K., 2008, "The 15-Year Cumulative Incidence of Retinal Vein Occlusion. The Beaver Dam Eye Study," Arch. Ophthalmol., 126(4), pp. 513-518.

[2] Klein, R., Peto, T., Bird, A., and Vannewkirk, M., 2004, “The Epidemiology of Age-Related Macular Degeneration,”Am. J. Ophthalmol., 137(3), pp. 486-495.

[3] Moss, S. E., Klein, R., and Klein, B. E. K., 1998, "The 14-Year Incidence of Visual Loss in a Diabetic Population," Ophthalmology, 105, pp. 998-1003.

[4] Jagtap, A. D., and Riviere, C. N., 2004, "Applied Force during Vitreoretinal Microsurgery with Handheld Instruments," Proc. IEEE Int. Conf. Eng. Med. Biol. Soc., pp. 2771-2773.

[5] Gupta, P. K., Jensen, P. S., and de Juan, E., 1999, Surgical Forces and Tactile Perception During Retinal Microsurgery, Springer, Berlin/Heidelberg, Germany.

[6] Jensen, P. S., Grace, K. W., Attariwala, R., Colgate, J. E., and Glucksberg, M. R., 1997, "Toward Robot-Assisted Vascular Microsurgery in the Retina," Graefe's Arch. Clin. Exp. Ophthalmol., 235(11), pp. 696-701.

[7] Charles, S., Das, H., Ohm, T., Boswell, C., Rodriguez, G., Steele, R., and Istrate, D., 1997, "Dexterity-Enhanced Telerobotic Microsurgery," Proceedings of 8th International Conference on Advanced Robotics, Monterey, California, Jul 7-9, pp. 5-10. 
[8] Nelson, B. J., Kaliakatsos, I. K., and Abbott, J. J., 2010, "Microrobots for Minimally Invasive Medicine,” Ann. Rev. Biomed. Eng., 12, pp. 55-85.

[9] Mitchell, B., Koo, J., Iordachita, I., Kazanzides, P., Kapoor, A., Handa, J., Hager, G., and Taylor, R., 2007, "Development and Application of a New Steady Hand Manipulator for Retinal Surgery," Proc. IEEE Int. Conf. Robot. Autom., pp. 623-629.

[10] Fleming, I., Balicki, M., Koo, J., Iordachita, I., Mitchell, B., Handa, J., Hager, G., and Taylor, R., 2008, Cooperative Robot Assistant for Retinal Microsurgery, Springer, Berlin/Heidelberg, Germany.

[11] Riviere, C. N., Ang, W. T., and Khosla, P. K., 2003, "Toward Active Tremo Canceling in Handheld Microsurgical Instruments," IEEE Trans. Rob. Autom., 19(5), pp. 793-800.

[12] Wei, W., Goldman, R., Fine, H., Chang, S., and Simaan, N., 2009, "Performance Evaluation for Multi-Arm Manipulation of Hollow Suspended Organs," IEEE Trans. Rob., 25(1), pp. 147-157.

[13] Ergeneman, O., Bergeles, C., Kummer, M. P., Abbott, J. J., and Nelson, B. J., 2010, "Wireless Intraocular Microrobots: Opportunities and Challenges," Surgical Robotics: Systems, Applications, and Visions, Springer US, Germany.
[14] Kummer, M., Abbott, J. J., Kratochvil, B. E., Borer, R., Sengul, A., and Nelson, B. J., 2010, "Octomag: An Electro-Magnetic System for 5-DOF Wireless Micromanipulation,” IEEE Trans. Rob., 26(6), pp. 1006-1017.

[15] Weiss, J. N., and Bynoe, L. A., 2001, "Injection of Tissue Plasminogen Activator into a Branch Retinal Vein in Eyes with Central Retinal Vein Occlusion," Ophthalmology, 108(12), pp. 2249-2257.

[16] Becker, N., and Batman, C., 2008, "Surgical Treatment of Central Retinal Vein Occlusion," Acta Ophthalmol., 86(3), pp. 245-252.

[17] Leng, T., Miller, J. M., Bilbao, K. V., Palanker, D. V., Huie, P., and Blumenkranz, M. S., 2004, "The Chick Chorioal-Lantoic Membrane as a Model Tissue for Surgical Retinal Research and Simulation," Retina, 24(3), pp. 427-434.

[18] Becker, B. C., Voros, S., MacLachlan, R. A., Hager, G. D., and Riviere, C. N., 2009, "Active Guidance of a Handheld Micromanipulator using Visual Servoing," Proc. IEEE International Conference on Robotics and Automation (ICRA), Kobe, Japan, pp. 339-344.

[19] Fung, Y. C., 1993, Biomechanics-Mechanical Properties of Living Tissues, Springer, New York, USA. 